\title{
OPTIONS WITH UNDERLYING ASSET DRIVEN BY A FRACTIONAL BROWNIAN MOTION: CROSSING BARRIERS ESTIMATES
}

\author{
GIULIA ROTUNDO \\ Department of Business, Technological and Quantitative Studies, Faculty of Economics, \\ University of Tuscia, via del Paradiso 47, 01100 Viterbo, Italy \\ and \\ Department of Mathematics for Economics, Financial and Insurance Decisions, Faculty of \\ Economics, University of Rome "La Sapienza", via del Castro Laurenziano 9, 00162 Rome, Italy \\ giulia.rotundo@uniroma1.it \\ ROY CERQUETI \\ Department of Mathematics for Economics, Financial and Insurance Decisions, Faculty of \\ Economics, University of Rome "La Sapienza", via del Castro Laurenziano 9, 00162 Rome, Italy \\ roy.cerqueti@uniroma1.it
}

Received March 25, 2006

Revised March 25, 2006

\begin{abstract}
This paper aims at supplying a decision support system tool to investors having options written on an underlying asset driven by a fractional Brownian motion (fBm). The results presented here rely on the theory of nonlinear transformations of $\mathrm{fBm}$ and provide the calculus of the probability estimate that the underlying asset crosses non linear barriers. Recent results stating a Black and Scholes-like pricing formula allow to monitor the expected behaviour of options on the basis of the dynamics of the underlying asset. The theory of speculative bubbles due to endogenous causes provides a useful suggestion for the detection of periods in which these results should be used. The application of the above results is shown through the NASDAQ case study.
\end{abstract}

\section{Introduction}

The typical problem of an agent taking position in a financial market is joint to the evaluation of the price of the underlying assets of her options. Large oscillations can change dramatically the returns at the expiry dates. It is interesting to know the most probable time which either the lowest or the highest values are reached at. Even if the excessive upwards or downwards that can also give rise to the suspension of the asset from the market could be considered in the realm of extreme events, automatic trading systems can trigger portfolio rebalancing and adjust hedging strategies when the option crosses some barrier. The study of the behavior of options relies on the study of the motion of the underlying assets. Fractional Brownian motion has been detected around a wide variety of financial data: 
stock market indexes, shares, foreign exchange currencies ${ }^{3,14,21}$. Moreover it was proven that the so called Ito type fractional Black-Scholes market has no arbitrage and is complete ${ }^{18,22}$. The Black and Scholes formula has been extended in order to calculate the fair price of an option when the underlying asset is driven by a geometric fractional Brownian motion ( $\mathrm{gfBm})^{11,31,32}$. So the problem of the evaluation of an option over an asset that crosses some barrier can be studied through the problem of the crossing of thresholds by a $\mathrm{fBm}{ }^{12,23,28}$. This is an intriguing problem that relies over bounds that connect $\mathrm{fBm}$ to Brownian motion, scaled Brownian motion, filtered Brownian motion ${ }^{20,30}$. The aim of this paper is thus to explore the open problem about the case of non linear barriers ${ }^{10}$ through the results about the transformation of the fractal degree of a process when filtered by a suitable function. The Brownian motion is always included as a particular case.

The outline of the paper is as follows. The next section resumes the main tools that can be used for the estimate of the probability of crossing linear barriers. Section 3. furnishes theoretical results in order to deal with some kind of nonlinear barriers. Section 4. provides at first a general discussion about the occurrence of the hypotheses that allow us to apply the above theory, and refers to the NASDAQ crash of April 2000 as a case study.

\section{Main theoretical tools}

This section aims to resume estimates of the probability of the first passage time for $\mathrm{fBm}$ with respect to the level of the barrier and to the time of crossing.

\subsection{Probability bounds for $\mathrm{fBm}$}

Let $B_{H}(t)$ be the $\mathrm{fBm}$ with parameter $H$. For $1 / 2<H<1, B_{H}(t)$ exhibits long term persistence and memory, whilst anti-persistence is characterized by $0<H<$ $1 / 2$. The particular case $H=1 / 2$ corresponds to Brownian motion ${ }^{25,29}$. Let $X(t)$ be either a $\mathrm{fBm} B_{H}(t)$ or a scaled Brownian motion $S_{H}(t)=B_{\frac{1}{2}}\left(t^{2 H}\right)$, and define

$$
A(X, c):=\sup \{X(t)-c t \mid t \geq 0\} .
$$

The following results hold ${ }^{7}$ :

Theorem 2.1. $\forall u \geq 0$ it results

$$
\begin{aligned}
& P\left(A\left(B_{H}, c\right)>u\right) \leq P\left(A\left(S_{H}, c\right)>u\right), 1 / 2 \leq H<1 \\
& P\left(A\left(B_{H}, c\right)>u\right) \geq P\left(A\left(S_{H}, c\right)>u\right), 0 \leq H<1 / 2 .
\end{aligned}
$$

The distribution of the suprema of a Brownian motion with drift obeys the law ${ }^{5}$

$$
P\left(A\left(B_{1 / 2}, c\right)>u\right)=\exp (-2 c u), \quad \forall u \geq 0 .
$$


An analogous result provides bounds for the probability that a $\mathrm{fBm}$ with drift crosses a fixed level $u$ in the scaled Brownian motion case ${ }^{7}$ :

$$
P\left(A\left(S_{H}, c\right)>u\right) \geq \exp \left(-2 a u^{2-2 H}\right), \quad \forall u \geq 0, a=\frac{1}{2}(c / H)^{2 H}\left(\frac{1}{1-H}\right)^{2-2 H}
$$

\subsection{First return time probability}

Let $T$ be the first return time of a fBm. Then

$$
P(T) \sim T^{H-2}
$$

Strictly speaking, for a continuous-time $\mathrm{fBm}$, the distribution of the first return time is not well defined in the sense that a finite time interval may contain infinite many crossing points with the zero axis and the above relation is valid in the limit of finite cutoffs that are always present in actual experiments due to the discrete nature of any measure apparatus ${ }^{8,26}$.

\subsection{Nonlinear Transformations of Fractionally Integrated Processes}

Long term memory properties depend strictly on the ordering of data. As an example operations like shufflings of data destroy the correlation structure. However nonlinear transformations of long memory processes through functions for which the Hermite rank $J$ can be calculated allow us to give results over the fractal measure of the resulting process ${ }^{10}$.

Theorem 2.2. Let $X(t)$ an $I(d)$ process, $H=d+1 / 2$, and let $g(\cdot): R \rightarrow R$ a function with Hermite rank equal to $J$. Then

(1) if $-1 / 2<d \leq 0$ then $g(X(t))$ is a short term memory process $I(0)$.

(2) if $0<d<1 / 2$ then $g(X(t))$ is $I(\bar{d}), \bar{d}=\max \{0,(d-.5) J+.5\}$

We recall that $g(X(t))=X(t)^{3}$ has Hermite rank $J=1 ; g(X(t))=X(t)^{2}$ and $g(X(t))=X(t)^{4}$ have both Hermite rank $J=2$.

\subsection{Fractional option pricing}

Let us consider an option which underlying asset follows a geometric fractional Brownian motion evolution. We have to introduce the fractional Black Scholes model (see ${ }^{13}$ for further considerations). In this setting, the discounted stock price is given by the Wick Ito Skorohod equation

$$
\delta S(t)=S(t) \mu(t) \mathrm{d} t+S(t) \sigma \mathrm{d} B_{H}(t)
$$

where $B_{H}(t)$ is a fBm under the probability measure $P$ and $\delta$ represents the divergence Malliavin operator. The solution of (2.7) is

$$
S(t)=S_{0} \exp \left(\int_{0}^{t}\left(\mu(s)-\sigma^{2} H s^{2 H-1}\right) \mathrm{d} s+\sigma B_{H}(t)\right)
$$


The Fractional Black-Scholes formula states that the price at every time $t \in[0, T]$ of an European call option with strike price $K$ and maturity $T$ is given by ${ }^{22,24}$

$$
C(t, S(t))=S(t) N\left(d_{1}\right)-K e^{-r(T-t)} N\left(d_{2}\right)
$$

where

$$
d_{1,2}=\frac{\ln (S(t) / K)+r(T-t) \pm \sigma^{2} / 2\left(T^{2 H}-t^{2 H}\right)}{\sigma \sqrt{T^{2 H}-t^{2 H}}}
$$

and $N(\cdot)$ is the cumulative probability of the standard normal distribution. Thus the mapping of $S(t)$ into $C(t, S(t))$ provides a direct relationship among the dynamics of $S(t)$ and $C(t, S(t))$.

\section{Barriers}

Let us consider a barrier $b(t)$. We are interested in determining the analytic form of nonlinear barriers for which it is possible to prove some theoretical result about the probability that $S(t)>b(t)$. The nonlinear dependence of $C(t, S(t))$ on $S(t)$ does not lead to a simple analytical formulation of the bounds directly on option prices, but the task can be performed by numerical analysis tools. In the stationary case (2.8) becomes

$$
S(t)=S_{0} \exp \left(\sigma B_{H}(t)+\mu t-\frac{1}{2} \sigma^{2} t^{2 H}\right)
$$

Let us consider a first case that uses directly the results of Theorem (2.1), and then other cases that need Theorem (2.2) before applying Theorem (2.1)

(1) If

$$
b(t)=\exp \left((\sigma c+\mu) t-.5 \sigma^{2} t^{2 H}+v\right)
$$

and $\left.u=\left(v-\ln \left(S_{0}\right)\right) / \sigma\right)$ then the problem $S(t)>b(t)$ is reduced to $B_{H}(t)>$ $c t+u$ and thus the results on the crossing of linear barriers by a $\mathrm{fBm}$ provided in Theorem (2.1) can be used.

(2) Let $J$ be the Hermite rank of $g(\cdot)$. Keeping the due attention to the monotone region of $g(\cdot)$ the problem of $B_{H}>\tilde{b}(t)$ can be solved through the study of $g\left(B_{H}\right)>g(\tilde{b}(t))$ if $g(\tilde{b}(t))=c t+u$ and if $g\left(B_{H}\right)$ is still a self similar process. According to Theorem (2.2), if $1 / 2<H<1$ and $\bar{d}=(d-.5) J+.5>0$ then $g\left(B_{H}\right)$ is a $\mathrm{fBm}$ with $\bar{H}=\bar{d}+1 / 2$. This result allows us to analyze the functions $g(\cdot)$ that have an Hermite rank.

(a) $g(X(t))=X(t)^{3}, J=1$. The condition $S(t)>b(t)$ leads to the following expression for the barrier:

$$
b(t)=\exp \left(\mu t-\frac{1}{2} \sigma^{2} t^{2 H}+\log \left(S_{0}\right)+\sigma(c t+u)^{1 / 3}\right)
$$

This transformation keeps the long memory property for each $\mathrm{fBm} B_{H}$ with $H>1 / 2$. 


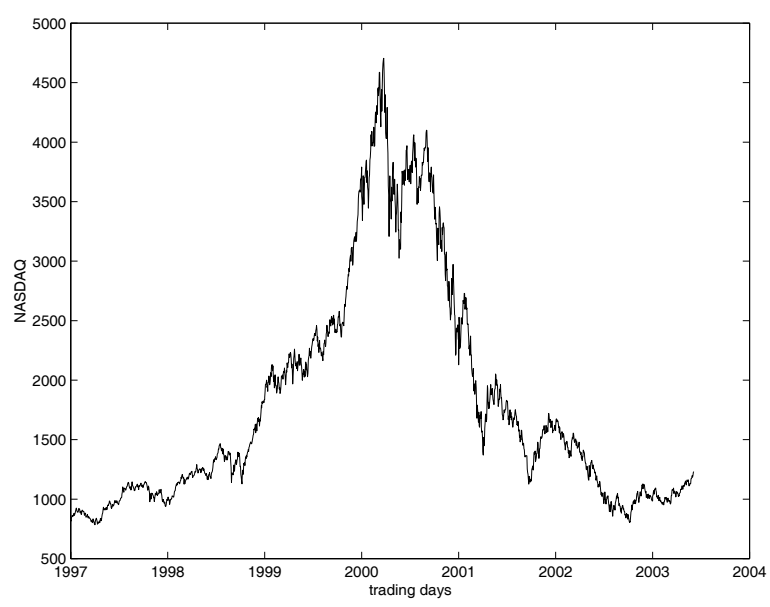

Fig. 1. The NASDAQ 100 Composite index from January 1st, 1997 to June 5th, 2003

(b) $g(X(t))=X_{t}^{2}, g(X(t))=X(t)^{4}, J=2$. In order to get a long term memory process $H>3 / 4$.

(c) $g(X(t))$ has an Hermite rank $J \geq 3$. In order to get a long term memory process $H>1 / 2+1 / 3$. The highest the Hermite rank, the highest $H$, and in general $d>\frac{1}{2}\left(1-\frac{1}{J}\right)$. Under this condition $\bar{d}>0$, thus the transformed process continues to be a long term memory process.

Moreover we can remark that the result (2.6) always applies and it provides a direct estimation of the probability of crossing of a fBm to cross horizontal barriers $g(b(t))=b$. Thus it can be applied in the case of the barrier (3.12) if $c=0$.

\section{Numerical results}

The theory shown in the previous sections can be used in order to provide the probability of crossing barriers if the underlying asset obeys a gfBm. Thus the first step towards the application of such analysis is the detection of gfBm. The theory about the speculative bubbles due to endogenous causes can provide a useful hint on the detection of periods of super than exponential growth ${ }^{33,15,34}$. The first common suggestion is to use the logarithm of market index data for the analysis in the case in which the magnitude of the crash is proportional to the price, as it happens for the NASDAQ crash of April $2000{ }^{16}$. Moreover the basic structure of (2.8) without taking into account the stochastic term shows a polynomial filtered by an exponential function. This constitutes a remarkable point toward the assessment of gfBm during the rise of a bubble due to endogenous causes because a polynomial approximation to the trend of the logarithm of data w.r.t. the time-to-crash given by $A+B\left(t_{c}-t\right)^{m}$ has been well explained by the theory and tested on data. In the NASDAQ 100 Composite case the values for the parameters $A=9.36(8.62,9.76)$, 

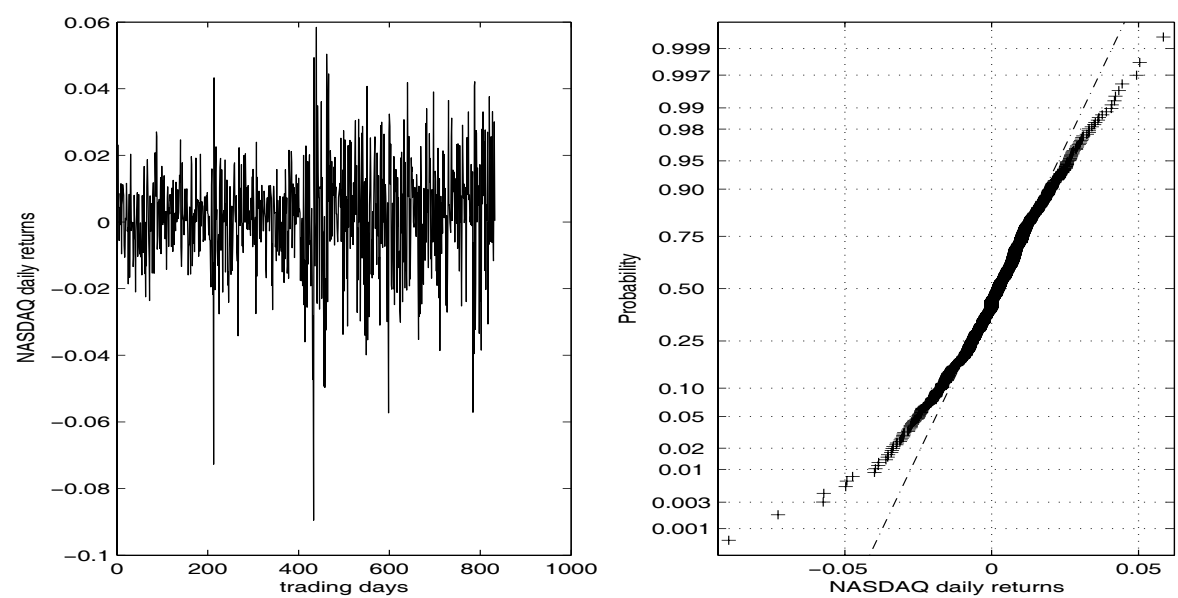

Fig. 2. Returns of the NASDAQ index since Jan. 1st, 1997 till April 11th, 2000 and normal distribution hypothesis testing. Both Lilliefors and Jarque-Bera tests are significant at $5 \%$ level, and reject the normal distribution hypothesis

$B=-1.60(-1.9,-0.89), m=0.26(0.18,0.36)$ and $t_{c}$ corresponding to April 11th, 2000, with a confidence interval ranging since March 30th, 2000, to April 24th, 2000 , are estimated by the minimum least squares method ${ }^{27}$ on a time series since Jan. 1st, 1997 till March 10th, 2000, containing a total amount of 833 daily data points. If the attention is focused on the residual noise instead of the deterministic trend then it can be observed that in the gfBm the $\mathrm{fBm}$ is filtered through an exponential function, but exponentials do not keep the self similarity degree ${ }^{10}$. In order to investigate the occurrence of gfBm at first the distribution of the daily returns $\{\log (S(t) / S(t-1))\}_{t=1, T-1}$ has been studied ${ }^{1}$ as a proxy for the returns. Numerical estimates give the mean $\mu=1.8 e-04(0.6 e-04,2.7 e-04)^{\mathrm{a}}$, and the standard deviation $\sigma=1.57 e-02(1.50 e-02,1.65 e-02)$. Both the Lilliefors test ${ }^{6}$ and the Jarque-Bera test ${ }^{17}$ reject the normal distribution hypothesis. The discrete version of $(2.7)$ is ${ }^{1}$

$$
\log (S(t) / S(t-1))=\mu+\sigma \Delta B_{H}
$$

The fractional Gaussian noise (fGn) $\Delta B_{H}$ is self similar. On the above data the self similarity parameter is $H_{\Delta}=0.57(0.56,0.59)$. The Detrended Fluctuation Analysis has been used in order to estimate the self similarity parameter ${ }^{2,19}$.

Let us now examine the first case (3.12). Linear barriers over $B_{H}(t)$ are exponential barriers over $S(t)$. An example is shown in Fig. 3.

The bound over the Hermite rank $J$ limits the range of transformations that it is possible to apply, depending on the persistence degree. On our data we can take

${ }^{\text {a }}$ For each variable empirically estimated the numbers inside the parentheses are the $95 \%$ confidence interval. 


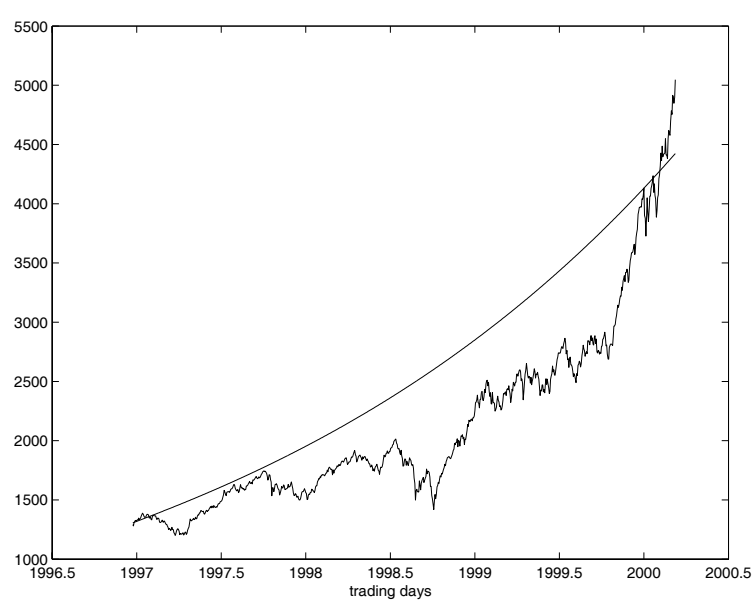

Fig. 3. The NASDAQ 100 Composite index from Jan. 1st, 1997, till March 10th, 2000. The curve is the barrier (3.12) for $c=.01$ and $u=0.63$

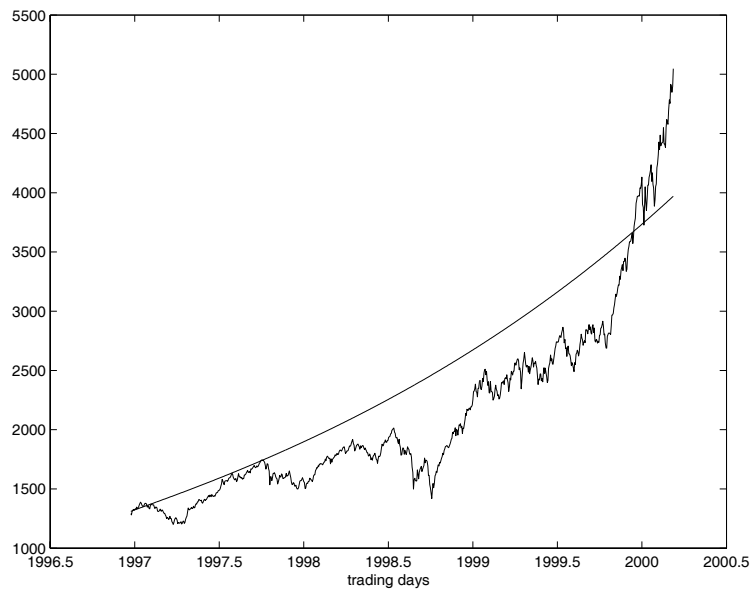

Fig. 4. The NASDAQ 100 Composite index from Jan. 1st, 1997, till March 10th, 2000. The curve is the barrier (3.13) drawn for the same values of $c=.01$ and $u=0.63$ used for the previous picture

into account the functions $g(\cdot)$ for which $J=1$, and as an example we show (3.13) in Fig. 4.

Each barrier depends on two parameters $c$ and $u$, and their values can be suggested by practical problems, like the measurement of the speed of data growth. They can be chosen in order to interpolate moving averages, which constitute part of technical analysis signals that can be used for triggering buy/sell orders. 


\section{Conclusions}

The paper explores the problem of an option crossing some barriers. The analysis is performed through the study of the underlying asset and by the reduction of this problem to the estimate of the crossing of barriers by $\mathrm{fBm}$. As a first approach the latter problem can be studied in the case of horizontal barrier by an immediate application of the first return time probability. This paper goes further on by showing how to use some results over the supremum of fractional Brownian motion and scaled Brownian motion with drift in order to perform a first step towards the probability description for the crossing of linear and non linear barriers. The task of non linear barriers can also give interesting insights over the structure of the market and take into account investor's set of preferences. The theory of speculative bubbles gives a hint on the detection of geometric fractional Brownian motion because of the polynomial super than exponential trend.

The symmetry between the ascending and the deflating part of speculative bubbles has been widely discussed through literature ${ }^{15}$ and in particular it has been tested for the NASDAQ 100 Composite index ${ }^{27}$. In this case the symmetry suggests to look for the occurrence of geometric fractional Brownian motion also during the deflate of the bubble. Moreover it has been shown that the main parameters that describe the bubbles belong to a short range of values. This result leads to a useful taxonomy of bubbles that provides a further hint on the detection of geometric fractional Brownian motion and hence to a wide application of the approach discussed through this paper, looking forward for the improvement of instrument for decision support systems and for the setting of automatic trading softwares.

\section{References}

1. Alexander, C., Market Models, John Wiley \& Sons Ltd., West Sussex, UK (2001)

2. Ausloos, M., Financial Time Series and Statistical Mechanics, in Computational Statistical Physics. From Billiards to Monte Carlo, eds. K. H. Hoffmann and M. Schreiber, Springer, Berlin (2001) 153-168

3. Ausloos, M. and K. Ivanova, Low $q$-moment multifractal analysis of gold price, Dow Jones Industrial Average and BGL-USD exchange rate, Eur. Phys. J., 8 (1999) 665669

4. Beran J., Statistics for Long-Memory Processes, Chapman \& Hall (1994)

5. Borodin, A. N. and P. Salminen, Handbook of Brownian Motion - Facts and Formulae, Birkhäuser Verlag, Basel (1996)

6. Conover, W. J., Practical Nonparametric Statistics, New York, Wiley (1980)

7. Debicki, K., Michna Z. and T. Rolski, On the supremum from Gaussian processes over infinite horizon, Probability and Mathematical Statistics, 18 (1998)

8. Ding, M. and W. Yang, Distribution of the first return time in fractional Brownian motion and its application fo the study of on-off intermittency, Phys. Rev. E, 52 (1995) 207-213

9. Embrechts, P. and M. Maejima, Self Similar Processes, Princeton Univ. Press (2002)

10. Dittmann, I. and C. W. J. Granger, Properties of Nonlinear Transformations of Fractionally Integrated Processes, UCSD Economics Discussion Paper No. 2000-07 (2000) 
11. Elliott, R. J. and J. van del Hoek, A general fractional white noise theory and applications to finance, Mathematical Finance, 13 No. 2 (2003) 301-330

12. Gripenberg, G. and I. Norros, On the prediction of fractional Brownian motion, J. Appl. Probab., 33, No. 2 (1996) 400-410

13. Haven E., An h-Brownian motion and the existence of stochastic option prices, Physica $A, 344$ No. 1-2 (2004) 152-155

14. Lo, A.W., Long memory in stock market prices, Econometrica, 59 (1991) 1279-1313

15. Johansen A. and D. Sornette, Endogenous versus Exogenous Crashes in Financial Markets, Contemporary Issues in International Finance, (In press)

16. Johansen. A. and D. Sornette, The Nasdaq crash of April 2000: Yet another example of log-periodicity in a speculative bubble ending in a crash, European Physical Journal B 17 (2000) 319-328

17. Judge, G. G., Hill, R. C., Griffiths, W. E., Lutkepohl, H. and T.-C. Lee, Introduction to the Theory and Practice of Econometrics, 2nd Ed., New York, Wiley (1982)

18. Hu, Y. and B. Oksendal, Fractional white noise calculus and application to Finance. Preprint, University of Oslo (2005)

19. Malamud, B. D. and D.L. Turcotte, Self-affine time series: measures of weak and strong persistence, Journal of Statistical Planning and Inference, 80 (1999) 173-196

20. Mandelbrot B. B. and J. W. Van Ness, Fractional Brownian Motions, fractional Noises and Applications, SIAM Review, 10 (1968) 422-437

21. Mantegna, R. N. and H. E. Stanley, Introduction to econophysics, Cambridge University Press, Cambridge (2000)

22. Necula, C., Option Pricing in a Fractional Brownian Motion Environment. Preprint.

23. Norros, I., A storage model with self-similar input, Queueing Systems Theory Appl., 16, No. 3-4, (1994) 387-396

24. Nualart, D., The Malliavin calculus and related topics, Probability and its Applications, Springer-Verlag, New York (1995)

25. Rangarajan, G. and M. Ding, Integrated approach to the assessment of long range correlation in time series data, Phys. Rew. E, 61 No. 5 (2000) 4991-5001

26. Rangarajan, G. and M. Ding, First passage time distribution for anomalous diffusion, Phys. Lett. A, 273 (2000) 322-330

27. Rotundo, G., Logistic function in large financial crashes, in The Logistic Map and the Route to Chaos: From the Beginning to Modern Applications, eds. M. Ausloos and M. Dirickx, Springer (2005)

28. Rotundo, G., The Hurst's exponent in technical analysis signals, in Practical Fruits of Econophysics: Proceedings of the Third Nikkei Econophysics Symposium, ed. H. Takayasu, Springer Verlag (2005)

29. Samorodnitsky, G. and M. S. Taqqu, Stable Non-Gaussian Random Processes, Chapman \& Hall, London (1994)

30. Sornette D., Helmstetter A., Endogeneous Versus Exogeneous Shocks in Systems with Memory, Physica A, (2003) $\mathbf{3 1 8} 577-591$

31. Sottinen, T. and E. Valkeila, On arbitrage and replication in the Fractional BlackScholes pricing model, Statistics \& Decisions, 21 (2003) 93-107

32. Sottinen, T., Valkeila, E., Fractional Brownian motion as a model in finance, Preprint, University of Helsinki, Department of Mathematics, 302 (2001)

33. Vandewalle, N., Ausloos, M., Boveroux, Ph., and A. Minguet, How the financial crash of October 1997 could have been predicted, Eur. Phys. J. B 4 (1998) 139-142 Rapid Notes

34. Vandewalle, N., Boveroux, Ph., Minguet, A., and M. Ausloos, The crash of October 1987 seen as a phase transition: amplitude and universality, Physica A: Statistical and 
10 GIULIA ROTUNDO AND ROY CERQUETI

Theoretical Physics, 255 No. 1-2 (1998) 201-210 